\title{
Cisplatin-induced change in moral conscience and behaviour
}

\author{
Jason Elzinga, Charles Jian \\ Faculty Reviewer: Peter Williamson, MD, PhD, FRCPC (Department of Psychiatry; Division of Neuropsychiatry)
}

\section{INTRODUCTION}

Cisplatin is a commonly used chemotherapeutic agent, which has been declared an essential medicine by the World Health Organization. ${ }^{1}$ Cisplatin was the first compound approved for use in what is now a class of platinum-containing chemotherapeutic drugs which exert their anticancer effect by cross-linking DNA, leading to cell death by apoptosis. ${ }^{2}$ Health Canada has approved cisplatin for use in treating advanced bladder cancer, metastatic ovarian cancer, and metastatic testicular cancer, but it is also commonly used to treat head and neck cancers, germ cell cancers, and small cell lung cancer. ${ }^{3}$ Like many chemotherapeutic drugs, cisplatin has many well documented side effects including nephrotoxicity, ototoxicity, and peripheral neuropathy. ${ }^{4}$

Neurotoxicity within the central nervous system (CNS) is not a recognized side effect of platinum-containing drugs. It has been suggested that the selectivity cisplatin demonstrates for damaging the peripheral nervous system is due to an inability of the drug to penetrate the blood brain barrier. ${ }^{5}$ This is due to the polar nature of platinum-containing compounds. A recent study in shrews showed that although platinum was detectable in the brain up to 72 hours after injection, the concentrations were at least 20 times lower than concentrations in plasma. ${ }^{6}$ This may help explain why CNS complications are not common with cisplatin use, even though peripheral neuropathy is seen in up to $50 \%$ of patients receiving treatment. ${ }^{7}$ However, a recently reported case by Barlinn et al suggested that cisplatin may in fact be able to cause CNS impairment in rare cases, and may have been responsible for major neurological and behavioural changes in a man undergoing cisplatin treatment for tonsillar cancer. ${ }^{8}$

\section{SUMMARY OF CASE REPORT ${ }^{8}$}

A 66-year-old man with tonsillar cancer was treated surgically with a tonsillectomy and radical neck dissection. Four weeks after the surgery, a regimen of targeted radiation and cisplatin chemotherapy was initiated. In the following 4 weeks of treatment with cisplatin, the patient displayed progressive personality changes including increased aggression, violence, and irritability. This resulted in the patient vandalizing his neighbour's house with an axe following an argument. The patient had also expressed suicidal thoughts to his wife. After threatening to push his wife down the stairs, police were called and the patient was ultimately brought to the hospital's emergency department.

The initial mental exam was unremarkable; the patient was properly oriented to time, place, and person. His demeanor was calm and cooperative. Additionally, there was no evidence of elevated mood or euphoria. He had no deficits in global cognitive functions. He had full recollection of his actions but did not express any remorse as he did not believe he had acted inappropriately. On history, the patient had been a chronic alcohol consumer until his cancer diagnosis, with no occurrence of alcohol withdrawal symptoms. No other pertinent medical or psychiatric history was reported. The family was adamant that this was not typical behaviour for the patient. Routine blood tests and brain computed tomography (CT) were unremarkable, and the patient was initially well-behaved. However, after perceived provocation, he became violent and destructive on the ward, destroying a radio which he believed was too loud, and assaulting a fellow patient and a nurse. The patient had to be restrained and treated with lorazepam and risperidone. The man remained unapologetic, insisting that his response was normal for a person who had been disturbed.

While admitted, chemotherapy was halted because of the "unpredictability" of the patient's behaviour, but radiation therapy continued. After stopping the cisplatin treatment, the patient's behaviour progressively improved, and 3 weeks after his last cisplatin dose, he returned to his original personality. Interestingly, he now expressed regret for his previous actions. He did not experience any memory loss, and was no longer irritable or violent.

\section{DISCUSSION}

The timing of symptom onset and recovery in this case strongly suggests cisplatin was responsible for the patient's neurological symptoms, demonstrating an extremely rare adverse effect of cisplatin treatment. There are no other reported cases of patients having cisplatin-induced aggression with moral conscience changes similar to the patient in this case. It has been shown that cisplatin can cause certain neurological complications. However, there are only a limited number of cases which report patients using platinum-containing drugs with solely psychiatric symptoms (ie, lacking any identifiable underlying conditions). ${ }^{9}$ These cases, although similar with regards to the psychiatric symptoms reported, have been diagnosed as both hypomania and acute psychosis..$^{10-13}$ All such cases had side effect onsets between 2 to 5 weeks after starting treatment with platinum-containing drugs. None occurred from the use of cisplatin therapy alone, but instead occurred in patients on multidrug regimens which including either cisplatin or carboplatin.

Although psychiatric changes induced by platinum-containing drugs are rare, neurological changes are not uncommon during chemotherapy with other cytotoxic agents. "Chemo brain" is one of the most frequently reported side effects of chemotherapy treatment for breast cancer, affecting up to $50 \%$ of patients. ${ }^{14}$ This condition is characterized by impairments in memory, attention, or other aspects of cognitive function, and its etiology is poorly understood. ${ }^{14}$ Neurological effects can also be much more severe including acute 
encephalopathy and seizures. Cisplatin is one of the many chemotherapy drugs which has been noted to cause posterior reversible encephalopathy syndrome (PRES), characterized by headache, seizures, confusion, and vision loss. ${ }^{9}$ Other anticancer drugs that have also been associated with PRES include methotrexate and enzalutamide, ${ }^{15,16}$ as well as both oxaliplatin and carboplatin. ${ }^{17}$

There are inherent difficulties with determining the cause of neurological symptoms in patients undergoing chemotherapy. Firstly, cancers are known to cause obscure neurological effects in some cases, even without chemotherapy. Neurological presentations of paraneoplastic syndromes are mediated by the immune system, which can affect the CNS of cancer patients without tumor invasion into the CNS. These syndromes can have a wide range of symptoms depending on the region of the brain affected. ${ }^{18}$ Thus, the symptoms of paraneoplastic syndromes can overlap with neurological symptoms of adverse chemotherapy reactions, making them difficult to differentiate. Secondly, many cancers are treated with fixed drug regimens, which can make it difficult to ascertain which drug is responsible for the neurological effect. For example, of the 3 cases of acute psychosis in patients treated with cisplatin, 2 of the patients were on the BEP regimen (bleomycin, etoposide, cisplatin), ${ }^{11,12}$ and one patient was treated with a combination of paclitaxel and cisplatin. ${ }^{13}$ One case suggested the psychosis was caused by the stress of having cancer and undergoing treatment in combination with other social factors, ${ }^{11}$ another attributed the psychosis to chemotherapy drugs without specifying which drug caused the effects, ${ }^{12}$ and the third believed that paclitaxel was responsible. ${ }^{13}$ The wide array of psychological effects which can be caused by cancers or the drugs used to treat them make it difficult to conclude that a chemotherapeutic agent was responsible for the symptoms.

Although there are inherent challenges with isolating chemotherapy drugs as the cause of neurological symptoms in cancer patients, especially if the onset of symptoms is gradual, it is important to keep drugs in the differential diagnosis until they can be ruled out. In the case by Barlinn et al, cisplatin therapy was stopped due to the uncooperative nature of the patient. It was not until the patient's behaviour improved that cisplatin was suspected as being responsible for the symptoms. ${ }^{8}$ The outcome of this case was positive, with the patient seeing a full return to his normal function. However, it seems that the patient's aggressive behaviour was the main reason cisplatin treatment was stopped. In future cases with CNS or psychiatric symptoms in a cancer patient, it is important to consider the possibility of drug reactions, since the patient may not be so aggressive that they are forced to discontinue chemotherapy.

\section{CONCLUSION}

This case of a cancer patient with changes in behaviour and moral conscience highlights not only a very atypical side effect of cisplatin therapy, but also some of the inherent difficulties in attributing CNS or psychiatric symptoms to chemotherapy drugs. In the present case, the patient's chemotherapy was stopped because of his uncooperative behaviour, and his symptoms improved dramatically. This case had a favorable outcome; the patient was discharged on risperidone but had generally recovered his personality. It is important that drugs be considered in the different diagnosis of chemotherapy patients with new onset symptoms. Many chemotherapeutic drugs have an array of associated side effects, with some being quite common, and others very rare. To recognize these rare "zebra" cases, one must be willing to think beyond the common causes, and consider what other possibilities could exist.

\section{REFERENCES}

1. World Health Organization. Geneva, CHE. WHO model list of essential medicines (19th Ed.) [Internet]. World Health Organization; 2015 Apr [cited 2016 Feb 1]. Available from: http://www.who.int/medicines/ publications/essentialmedicines/en/.

2. Dasari S, Tchounwou P. Cisplatin in cancer therapy: molecular mechanisms of action. Eur J Pharmacol. 2014 Jul;740:364-78.

3. Cisplatin Drug Monograph. Toronto (ON): Cancer Care Ontario Formulary; 2015 Feb. 11 p.

4. Hartmann J, Kollmannsberger C, Kanz L, Bokemeyer C. Platinum organ toxicity and possible prevention in patients with testicular cancer. Int J Cancer.1999 Dec;83(6):866-9.

5. Englander E. DNA damage response in peripheral nervous system: coping with cancer therapy-induced DNA lesions. DNA Repair. 2013 Aug;12(8):685-90.

6. Eiseman J, Beumer J, Rigatti L, Strychor S, Meyers K, Dienel S, et al. Plasma pharmacokinetics and tissue and brain distribution of cisplatin in musk shrews. Cancer Chemother Pharmacol. 2014 Jan;75(1):143-52.

7. Hoop R, Van Der Burg M, Huinink W, Houwelingen J, Neijt J. Incidence of neuropathy in 395 patients with ovarian cancer treated with or without cisplatin. Cancer. 1990 Oct;66(8):1697-702.

8. Barlinn K, Lehrach H, Siepmann T, Braeuer D, Buntrock U, Sassim N. Temporary loss of moral behavior in a patient undergoing chemotherapy with cisplatin - breaking bad. BMC Psychiatry. 2015 Feb;15(9).

9. Simkens G, Hanse M, Hingh I. Acute neurological disorders following intraperitoneal administration of cisplatin. Int J Gynaecol Obstet. 2012 Mar;120(3):291.

10. Matsunaga M, Onishi H, Ishida M, Miwa K, Araki K, Kaneta T, et al. Hypomanic episode during recurrent gastric cancer treatment: report of a rare case and literature review. Jpn J Clin Oncol. 2012 Oct;42(10):961-4.

11. Campbell B, Panicker J. New onset psychosis in an adolescent during treatment of testicular germ cell tumor. J Pediatr Hematol Oncol. 2011 Apr;33(3):125-6.

12. Puangthong U, Pongpirul K. Chemotherapy-induced acute psychosis in a patient with malignant germ cell tumour. BMJ Case Rep. 2015 Apr;2015.

13. Ahmed N, Usmani S, Jabbour N, Hegde U. Acute psychosis after paclitaxel infusion. Conn Med. 2011 Sep;75(8):465-6.

14. Staat K, Segatore M. The phenomenon of chemo brain. Clin J Oncol Nurs. 2005 Dec;9(6):713-21.

15. Aradillas E, Arora R, Gasperino J. Methotrexate-induced posterior reversible encephalopathy syndrome. J Clin Pharm and Ther. 2011 Aug;36(4):529-36.

16. Crona D, Whang Y. Posterior reversible encephalopathy syndrome induced by enzalutamide in a patient with castration-resistant prostate cancer. Invest New Drugs. 2014 Jun;33(3):751-4.

17. Skelton M, Goldberg R, O’neil B. A case of oxaliplatin-related posterior reversible encephalopathy syndrome. Clin Colorectal Cancer. 2007 Jan;6(5):386-8.

18. Martel S, Angelis F, Lapointe E, Larue S, Speranza G. Paraneoplastic neurologic syndromes: clinical presentation and management. Curr Probl Cancer. 2014 Jul-Aug;38(4):115-34. 